\title{
Analysis and Design of a Filestream Based English Language Learning System
}

\author{
Mohamed R. Mhereeg1*, Asma G. Tawil ${ }^{2}$ \\ ${ }^{1}$ Faculty of Information Technology, University of Tripoli, Tripoli, Libya. \\ 2 Faculty of Engineering Technology, Institute of Computing Technology, Janzoor, Tripoli, Libya. \\ * Corresponding author. Tel.: 00218918234600; email: mmhereeg@msn.com \\ Manuscript submitted March 5, 2015; accepted May 3, 2015. \\ doi: $10.17706 /$ jcp.10.4.268-283
}

\begin{abstract}
The paper discusses the feasibility of constructing a SQL Server FILESTREAM based English language learning system (ELLS). The paper focuses on the analysis and design phase of the system. It explains the prospect of storing and managing unstructured data (e.g. Images, video, Word, Excel, PDF, MP3, etc) for educational purposes using FILESTREAM technique provided by SQL Server 2012, and how to maintain efficient storage and access to BLOB data. However, Storing unstructured data posed many challenges, such as how to maintain transactional consistency between the structured and unstructured data, how to manage backup, restore, and storage performance. The paper seeks to utilise the combination of SQL Server 2012 features and the NTFS (new technology file system) to overcome these issues and attempt to improve the efficiency and performance of the ELLS for children. The system also seeks to maintain the transactional consistency between the unstructured data and corresponding structured data. Several UML analysis techniques have been used to illustrate the analysis of the system requirements such as the Use Case diagrams, Sequence diagrams, Activity diagrams and Class diagrams. The database design of the system is also presented in this paper.
\end{abstract}

Key words: BLOB, ELLS, filestream, NTFS, unstructured data.

\section{Introduction}

In recent years, there has been an explosion in the volume of digital data created and stored by both individuals and organizations [1] Historically, businesses have used computer systems and databases to store most of their business data in structured formats such as relational tables or fixed format files, and software applications have used these structured data stores to perform business tasks. Today however, a large proportion of an organization's data is typically stored in documents created with productivity tools such as Microsoft Office Excel and Microsoft Office Word, and advances in digital photography, document scanning, video production, and audio formats have further extended the range of unstructured data formats that are used for business data [1]. For example, in the context of relational database systems, it refers to data that can't be stored in rows and columns. Additionally, dramatic reductions in the cost of hardware storage and memory have significantly affected the amount and type of data that is stored in computer systems, and led to the emergence of a new generation of business application that merges traditional relational data structures with unstructured digital content [2].

This profusion of digital content means that organizations are now seeking to manage both relational data and unstructured data at the enterprise scale, and require a solution that comprehensively meets the 
needs of relational and non-relational data storage while reducing the cost of managing and building applications for that data [1].

Storing unstructured data such as text documents, images, and videos posed many challenges, such as how to maintain transactional consistency between the structured and unstructured data, how to manage backup and restore, and storage performance and scalability. Architects of applications that required the storage of binary large objects (BLOB) data could either store the data in the database or store it outside of the database with a reference stored in the database [2], [3].

This paper focuses on unstructured data and describes the FILESTREAM feature of SQL Server 2012, which allows storage of and efficient access to BLOB data using a combination of SQL Server 2012 and the NTFS (new technology file system). The study focuses also on the clarification the advantages and disadvantages of the use of this feature with choices for BLOB storage. An English language learning system has been developed in which this features has been applied. The system is intended to offer an excellent supporting material for in-class teaching, developing some language skills like reading, listening, and speaking at a certain level. The system will also allow doing tests and providing feedback to students.

\section{Requirements Analysis}

Requirement analysis involves understanding the problem, establishing the system and the constraints under which the system will operate. It is one of the very vital activities in the development of a thesis/project and all later stages depend on it [4]. There are two kinds of requirements in the system:

- Functional requirements

- $\quad$ Non-functional requirements

\subsection{Functional Requirements}

The system has two different types of system users: admin (Teacher) and student, each type of user has specific requirements which are described as the following

\section{- Administrator (Teacher)}

Login to the system, Add users, View users, Delete users, Edit users, Add level, View level, Delete level, Edit level, Add Question, View Question, Delete Question, Edit Question, Add subject, View subject, Delete subject, Edit subject, Add test/answer, View test/answer, Delete test/answer, Edit test/answer, Add Result, View Result, Add feedback, View feedback.

\section{- $\quad$ Students}

Login to the system, Change the password, View the numbers, View the letters, View the Lessons, View the results.

\subsection{Non Functional Requirements}

Security requirements are important factors in this system as classified data will be stored in the database. User validation will be done during login to insure that the user is valid and that the user only has access to his or her permitted data. General users will only have access through the user interface.

The system will have consistent interface formats and button sets for all forms in the application, the system will also have a form based interface for all data entry and viewing formats, and will generate reports that are formatted in a table that should look like the existing manual report formats for the users.

\section{Use Case Diagrams}

The Use Case Diagrams (UCD) is an Object-Oriented analysis tool has been used to present the functional requirements of the system and also to define the scope of the project. It has been used as a communication tool between the users and the functions of the systems to perform the transactions of the system. 


\subsection{Use Case Overview of the System}

The following diagram shown in Fig. 1, clarifies the use case requirements of the proposed system.

The administrator (Teacher) heads to the control panel after successful log-in to the system. In control panel, there are six sections available: User Management, level Management, question Management, Lessons Management, test/answer Management and Result Management. The student is permitted to use the user interface after a successful login to the system. There are four sections available for students: Change password, View lessons (Video, Listening), Do test, and View Results. Each section has many functions which belong to business logic layer. These functions are explained using the Use Case diagrams.

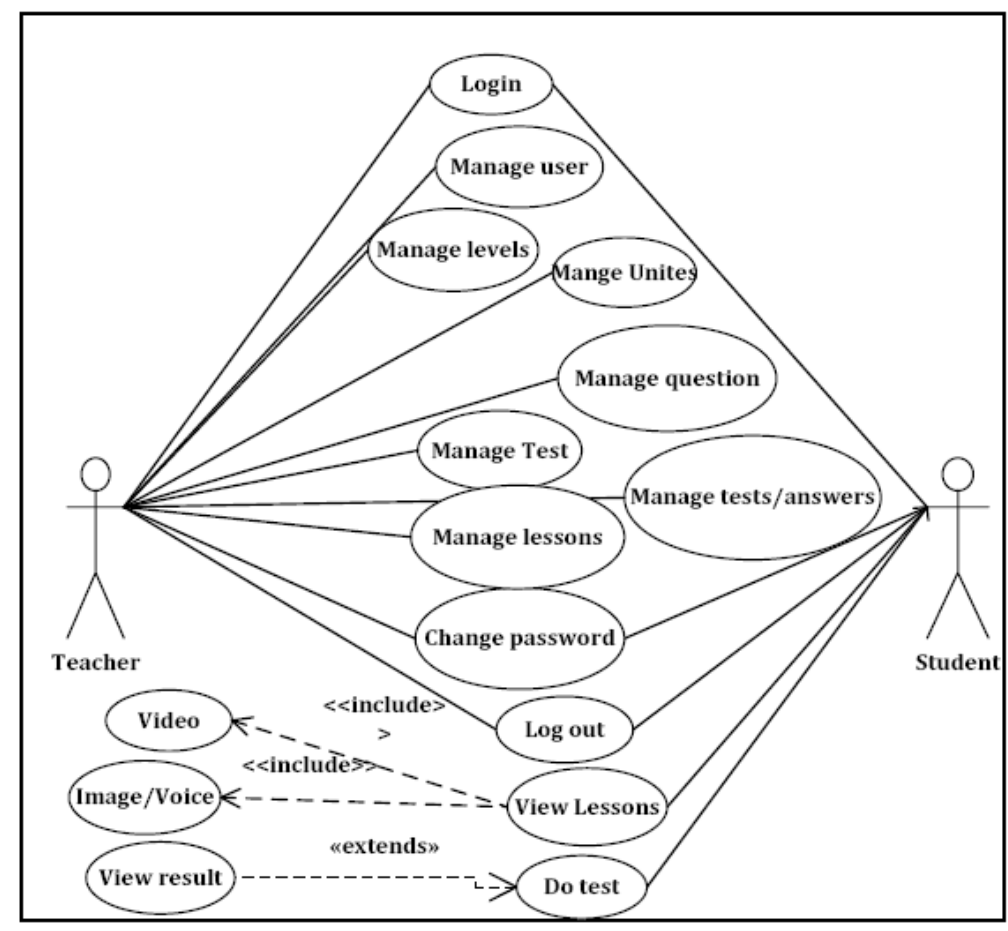

Fig. 1. Use case overview of the system.

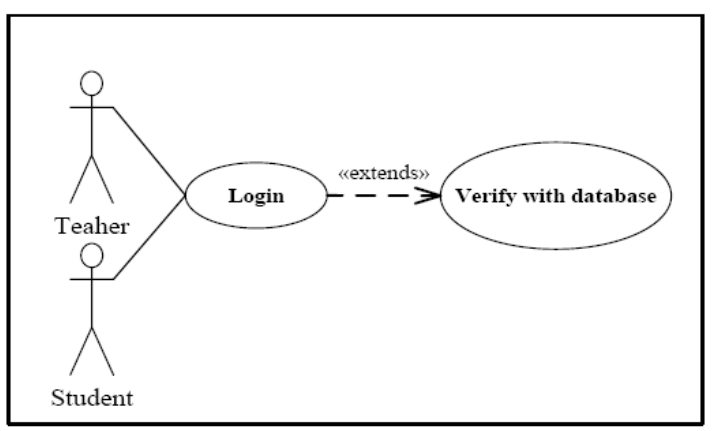

Fig. 2. Login use case.

\subsection{Login Use Case}

It describes the login access method used in the system for both users, Teacher and Student, each user is given a specific authorization according to the user type. System Administrator should be given a unique account to manage the system, if the user already has his own account, he/she can write his/her username and password to $\log$ on, then the application will verify the username and password information, if the information is true, then the user will be logged on to his/her account and then the user can do his own all operations available, but, if the information is incorrect, then the user will be asked to type them again correctly (see Fig. 2). 


\subsection{Manage Users Use Case Diagram}

After a successful login, the teacher can add, edit and delete students. Add, edit and delete levels, Questions, lessons (Video, Listening). Prepare tests /Answers and view results. The following diagram shown in Fig. 3, clarifies all use cases that allow creating new user, edit and delete user in the system by the teacher. An administrator (teacher) can add a new user, delete an old user, and modify user information. A new user can be added by calling "Create new user" function, followed by hitting the button "Save". Following the same manner, a user can be deleted by calling the "Delete user" function. The "Edit" function is a very important function, because the administrator calls "Edit user" function in order to modify the user's information.

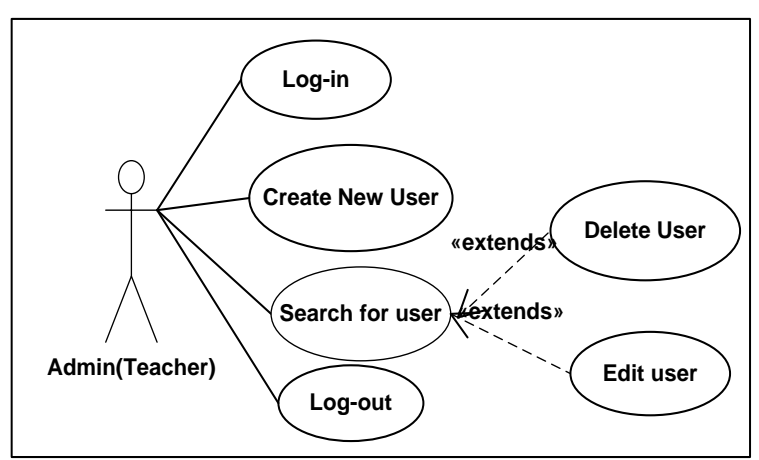

Fig. 3. Manage users use case diagram.

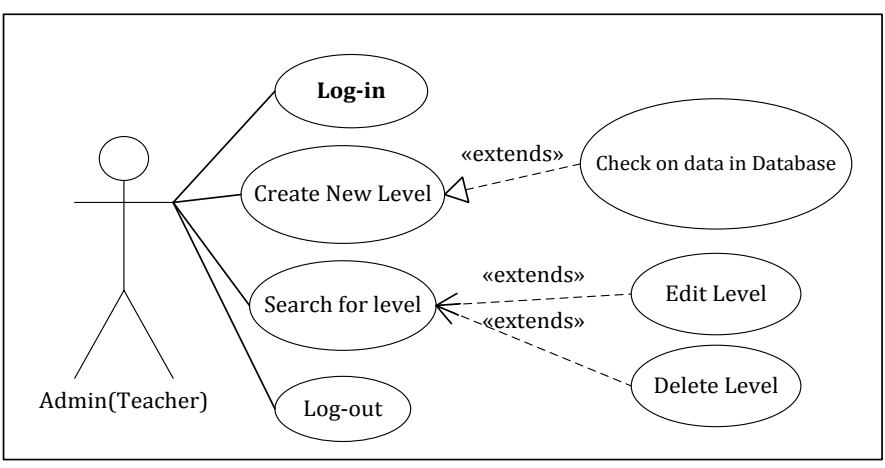

Fig. 4. Manage levels use case diagram.

\subsection{Manage Levels Use Case Diagram}

The above diagram shown in Fig. 4, clarifies all use cases that allow creating new level, edit and delete level in the system by the teacher. There are three functions available. An administrator (teacher) can add a new level, delete an old level, and modify level. The administrator can add a new level by calling "Create new levels" function, and doing a "save" action. He also can delete a level by calling the "Delete level" function. The "modify level" function" can be operated after the "search for level "function is called.

\subsection{Manage Question Use Case Diagram}

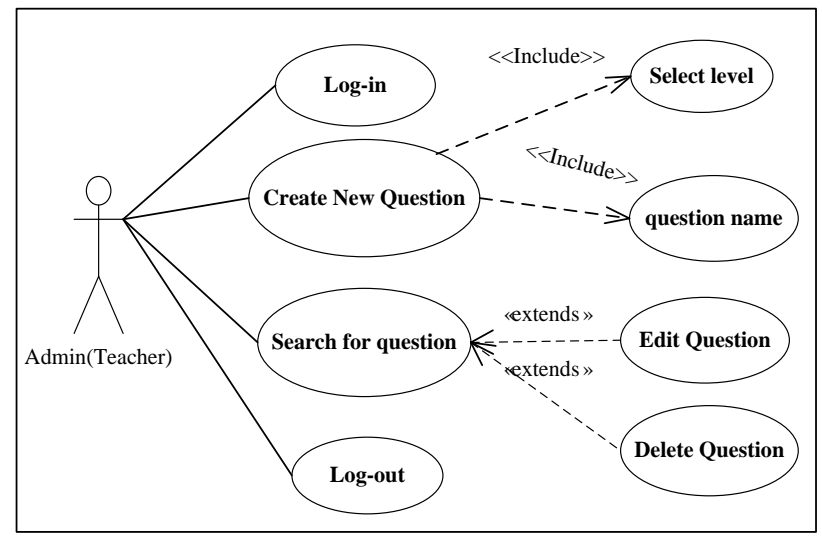

Fig. 5. Manage question use case diagram.

The above diagram shown in Fig. 5, clarifies all use cases that allow create, edit and delete questions in the system by the teacher. The administrator (teacher) can add a new question by calling "Create new question" function, and doing a "save" action. And he can delete a question by calling the "Delete question" function. The "modify question" function, can be operated after calling the "search for question "function is achieved. 


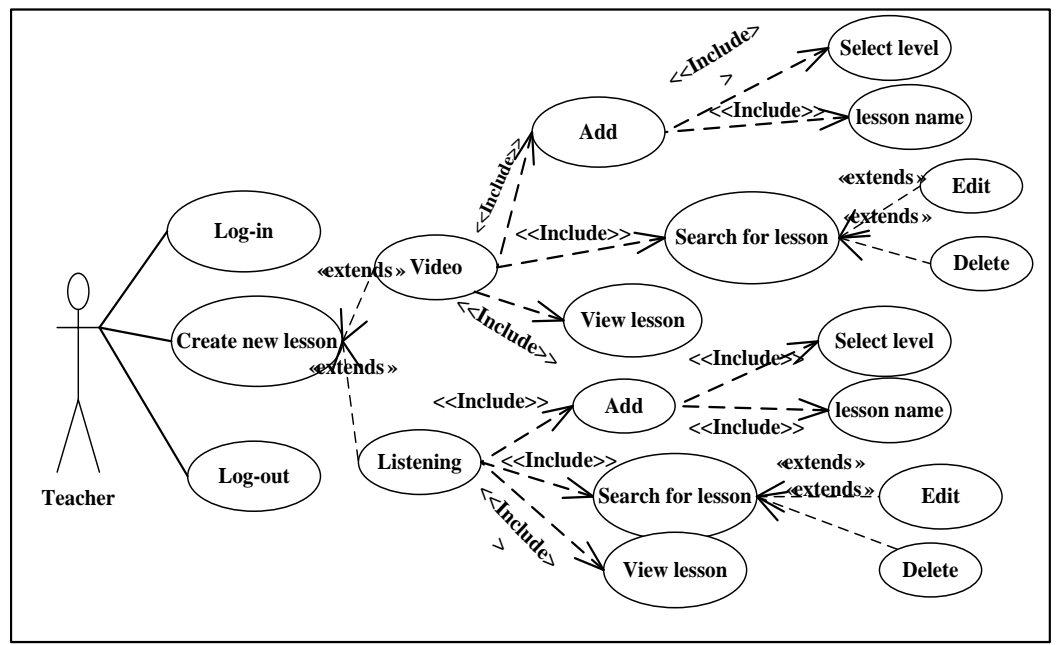

Fig. 6. Manage lessons use case diagram.

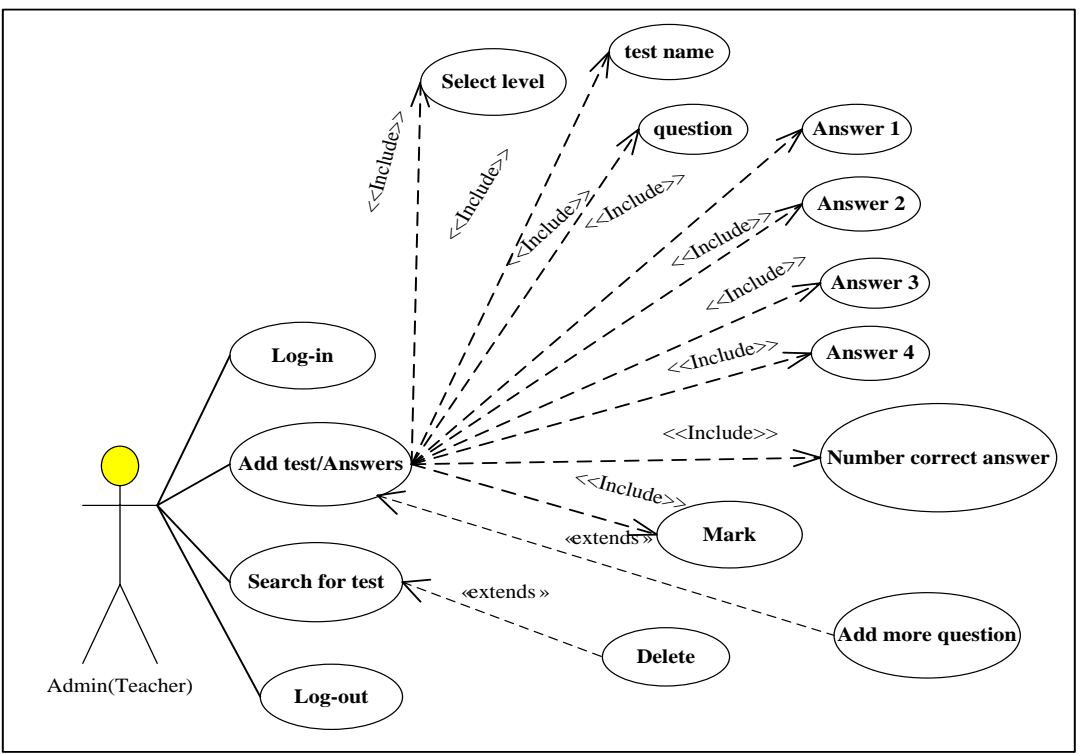

Fig. 7. Manage test/answer use case diagram.

\subsection{Manage Lessons Use Case Diagram}

The above diagram shown in Fig. 6, clarifies the two types of educational lessons used in the system, the use case (Create new lessons) is divided into two types of educational lessons, namely video and listening, each use case includes several functional requirements (add, edit and delete functions) as part of the functionality of the system.

Video: This section contains an explanation of a variety of lectures include (words, conversation, and grammar).

Listening: This section contains a variety of paragraphs include (sentences/words and pronunciations).

\subsection{Manage Test/Answer Use Case Diagram}

The above diagram shown in Fig. 7, clarifies all use cases that allow creating a new test, edit and delete tests in the system by the teacher. There are two functions available. An administrator can add a new test, and Delete test information. The administrator can also add a new test by calling "Create new test/answer" function. This function includes the use cases (select level, test name, question name, answer 1, answer 2, answer 3, answer 4, number the correct answer, mark and adding more questions), and then hitting the 
"save" button. The administrator can also delete a test by calling the "delete test/answer" function that a successful call to the "search for test/answer" function.

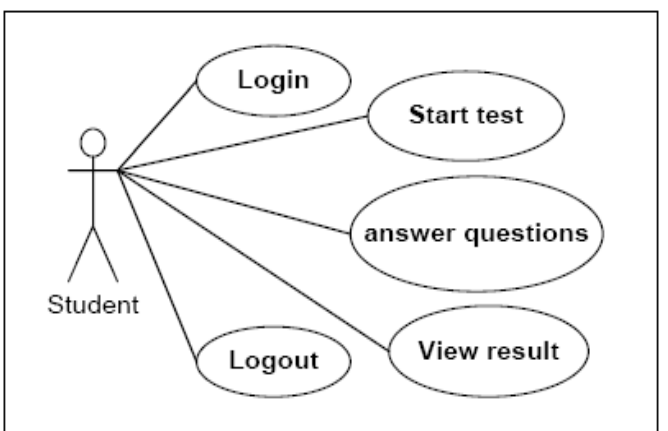

Fig. 8. Do test use case diagram.

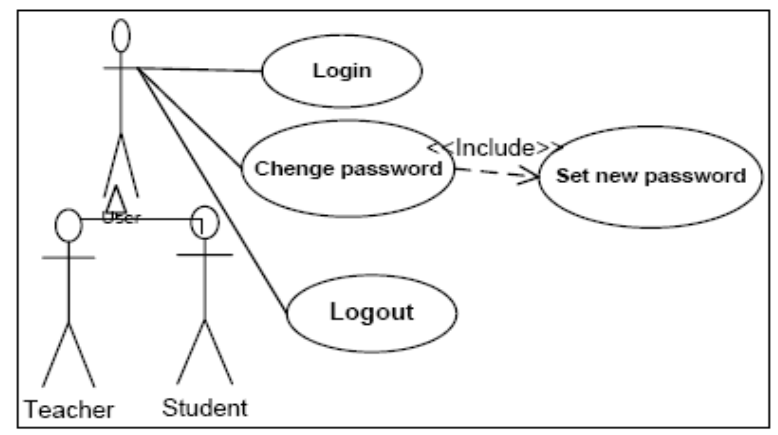

Fig. 9. Change password use case diagram.

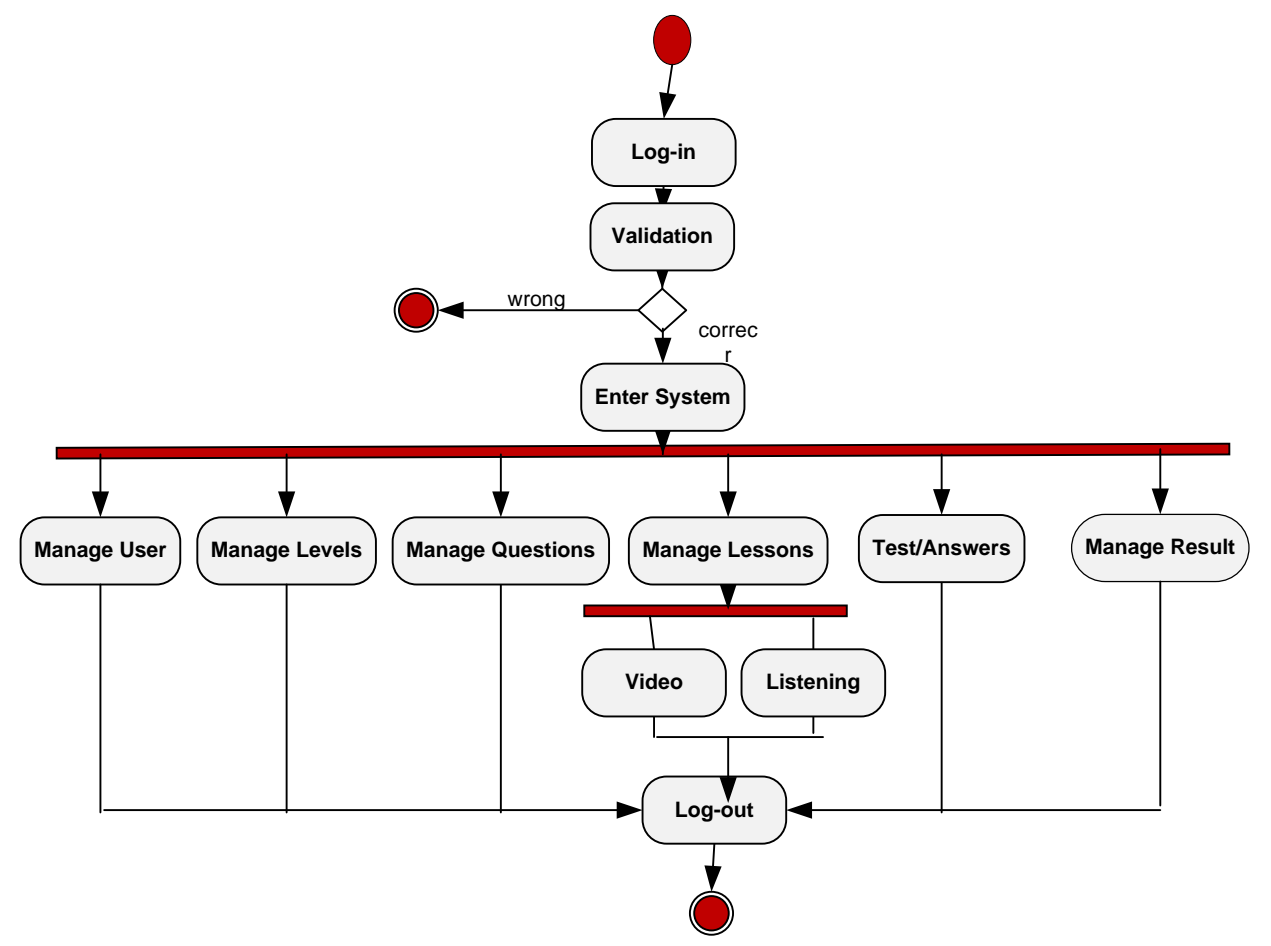

Fig. 10. Administrator (teacher) activity diagram.

\subsection{Do Test Use Case Diagram}

This diagram includes all use cases that allow taking the test in the system by the students, after students finish answering the questions, they can view the results (see Fig. 8).

\subsection{Change Password Use Case Diagram}

This diagram (Fig. 9) presents the use case that allows both system users the teacher and students to change the password to an appropriate one when needed.

\section{Activity Digram}

In Unified Modeling Language (UML) an activity diagram is used to display the sequence of activities. Activity diagrams show the workflow from a start point to the finish point detailing the many decision paths that exist in the progression of events contained in the activity. They may be used to detail situations where parallel processing may occur in the execution of some activities. Activity diagrams are useful for business modeling where they are used for detailing the processes involved in business activities [5]. 


\subsection{Administrator (Teacher) Activity Diagram}

After a successful login, the teacher will be able to create new student accounts, add levels, questions, test/answers to a particular lesson. The teacher has to choose how to save the lessons (Video or Listening).

If the teacher chooses to save the lessons as ready lessons that means the lesson will be ready to use, the student will be able to see these lessons when they enter the system. In other hand, the lessons saved as draft lessons, only the teacher who creates them can view, edit or delete these lessons (see Fig. 10).

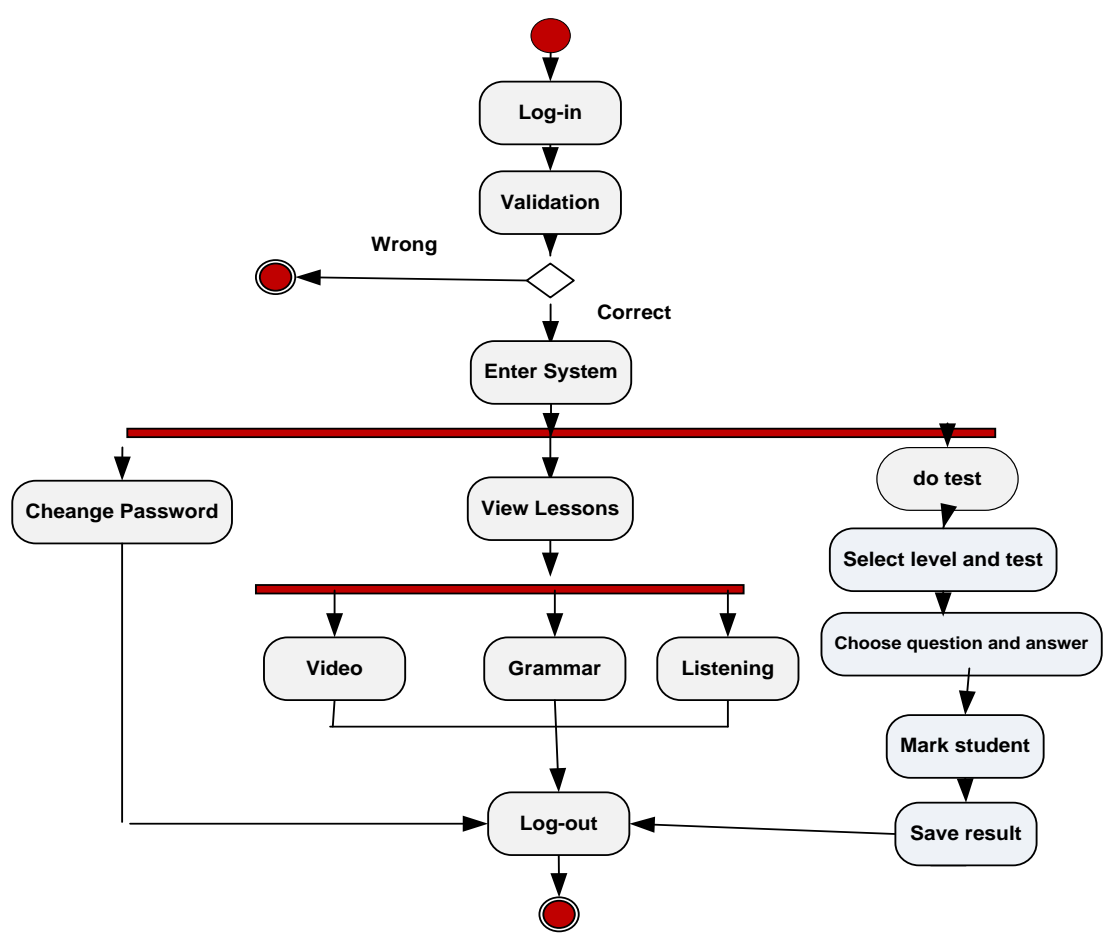

Fig. 11. Student activity diagram.

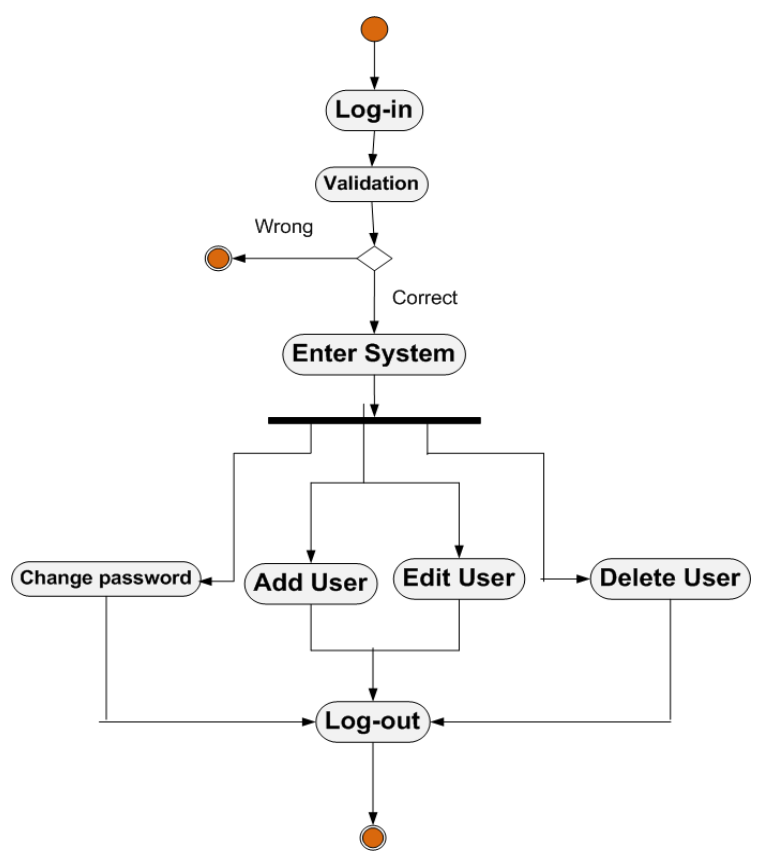

Fig. 12. Register user activity diagram.

\subsection{Student Activity Diagram}

When the students logs-in successfully he will be able to: 
1) Change password: Changing the password requires the username, old password, New Password, and then confirm the password.

2) Do a test and view the results: Student can hit select level and test to view the questions, after the completion of the test the result is displayed.

3) View lessons: Including all types of lessons watching, Reading and listening (see Fig. 11).

\subsection{Change Password User Activity Diagram}

When the teacher logs-in successfully he will be able to create new user, edit, delete user and change password (see Fig. 12).

\section{Class Diagram}

A class diagram is an illustration of the relationships and source code dependencies among classes in the unified modeling language (UML). The class used to define the methods and variables in an object, which is a specific entity in a program or the unit of code representing that entity. Class diagrams are useful in all forms of object-oriented programming (OOP). The following class diagram in Fig. 13 describes potential utility classes designed for this application. The first line on top of each class refers to the name of the class. The second segment on the stack represents the attributes of the class. The methods, which are listed on the third segment of the stack, indicate the class operation that can be used within or outside the class.

\subsection{Level Class}

This class is associated with the user table in the database and has utilities to manipulate data in this table such as selection, insertion, deletion, updating and getting information about the level.

\subsection{Question Class}

This class is associated with the test_question table in the database and has utilities to manipulate data in this table such as selection, insertion, deletion, updating and getting information about the question.

\subsection{Lesson_Listening Class}

This class is associated with the level table in the database and has utilities to manipulate data in this table such as selection, insertion, deletion, updating and getting information about the lessons.

\subsection{Lesson_Video Class}

This class is associated with the level table in the database and has utilities to manipulate data in this table such as selection, insertion, deletion, updating and getting information about the lessons.

\subsection{User Class}

This class is associated with the User_type table in the database and has utilities to manipulate data in this table such as selection, insertion, deletion, updating and getting information about the user.

\subsection{User_Type Class}

In this class the type of user are stored within the database to select the users Permissions.

\subsection{Test_Data Class}

This class is associated with the User table in the database and has utilities to manipulate data in this table such as insertion and getting information about the test and results.

\subsection{Result Class}

This class is associated with the Test_data table in the database and has utilities to manipulate data in this table such as insertion and getting information about the Results. 


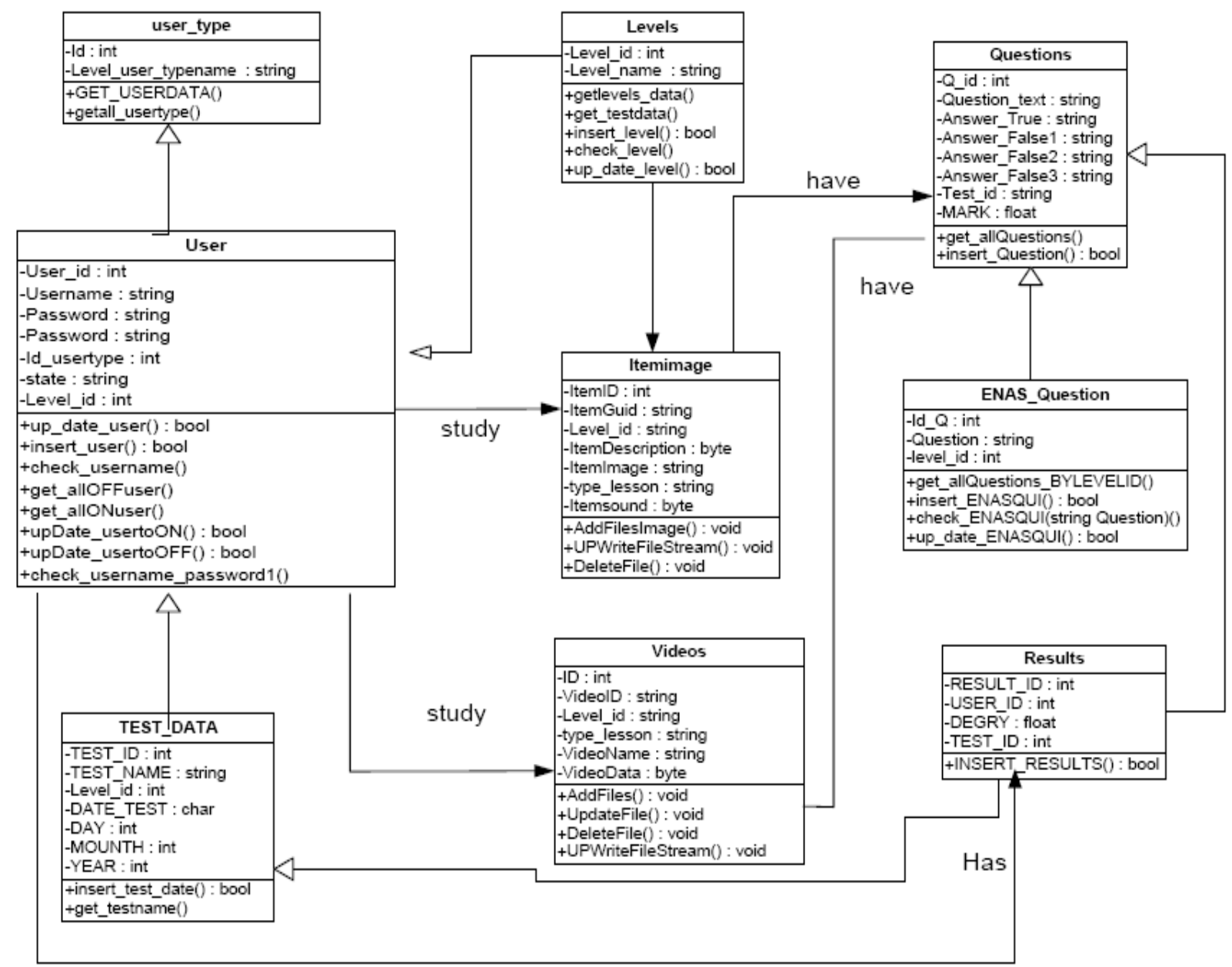

Fig. 13. Class diagram.

\section{Sequence Diagram}

The below diagram is a model describing how groups of objects collaborate in some behavior over time, the diagram captures the behavior of a single use case, it shows objects and the messages that are passed between the objects of the system. The sequence diagram has been used in this System to show the objects interaction as the following:

\subsection{Student Sequence Diagram}

Fig. 14 below illustrates the student sequence diagram. Sequence diagrams demonstrate the behavior of objects in a use case by describing the objects and the messages they exchange. The diagrams below shows an object of Student starts the behavior by sending a message to the Interface object. Messages pass between the different objects until the object User receives the final message.

1) Interaction between the student object and the user object through (Login to the system, validate the data and return the results of the verification).

2) Interaction between the student and the lessons (video) objects, through performing the basic functions (choose the lesson name, view lesson), including giving confirmation messages for each function exists.

3) Interaction between the student object and the lessons (Listening) object, through performing the functions (choose the lesson name, view lesson), plus giving confirmation messages for each function exists.

4) Interaction between the student object and the do test object through (choose test name, view question 
and answers), including giving confirmation messages for each function exists.

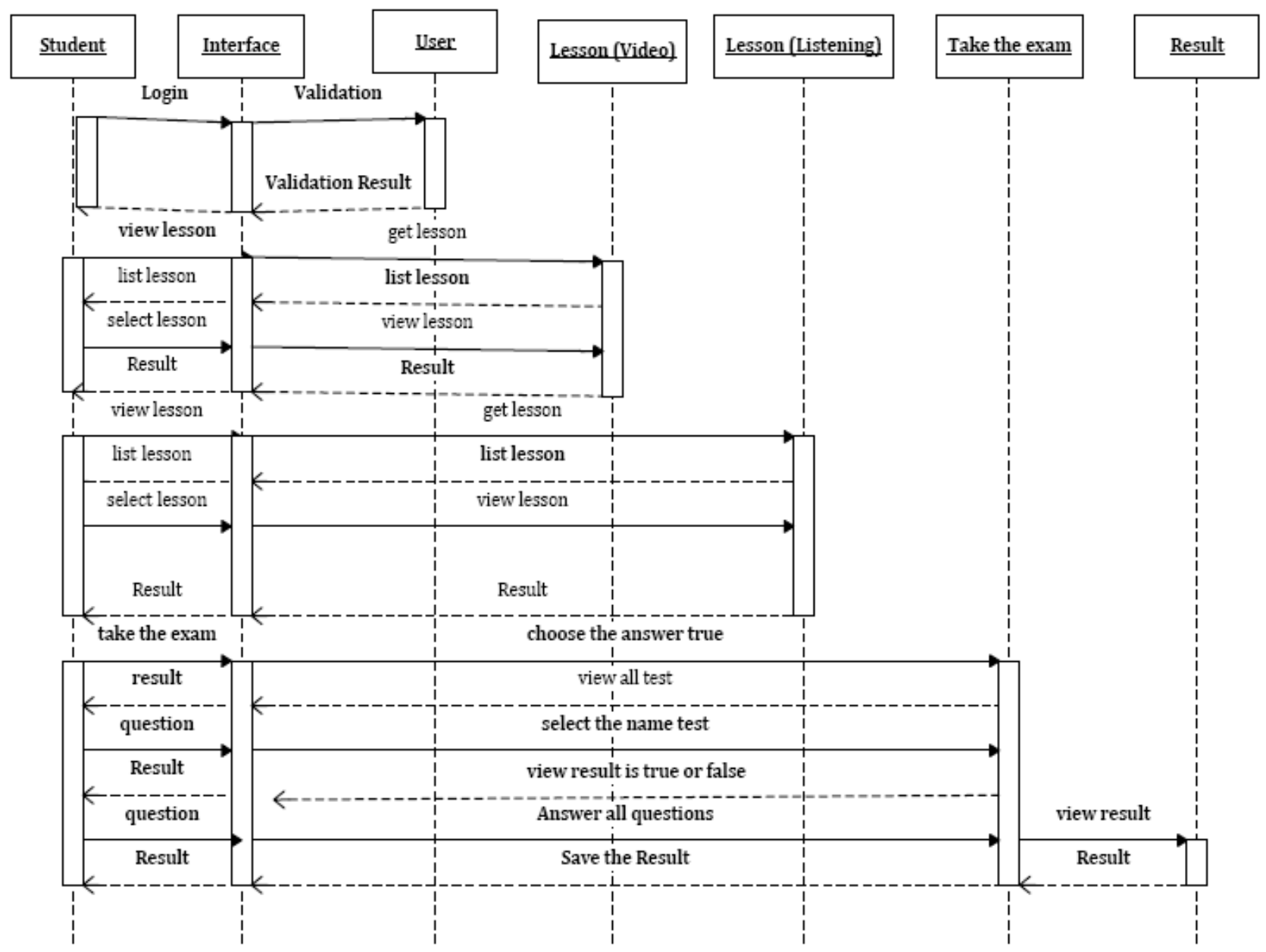

Fig. 14. Student sequence diagram.

\subsection{Admin (Teacher) Sequence Diagram}

The sequence diagram in Fig. 15 illustrates a series of sequential interactions between the teacher and participating objects into the system.

1) Interaction between the teacher object and the user object through (Login to the system, validate the data and return the results of the verification).

2) Interaction between the teacher object and the student object, through perform the basic functions from (add new student, view student, modify and delete).

3) Interaction between the teacher object and the levels object through (add new levels, view level, and modify previous data), with giving confirmation messages for each function exist.

4) Interaction between the teacher object and the question object through (add new question, view question, and modify previous data), with giving confirmation messages for each function exist.

5) Interactions between the teacher object and the lessons object through (add new lessons, view lessons, and modify previous data), with giving confirmation messages for each function exist.

6) Interactions between the teacher object and the test object through (add new test, view test, and modify previous data), with giving confirmation messages for each function exist.

\section{Database Design}

After the completion of the system analysis phase, it is time to step into the design phase. Since the data is 
analyzed, and the relationships between the data are accomplished, the E-R model is established, and then the concept structure and logic structure of database are designed. Because SQL Server 2012 can be utilized with high efficiency to a variety of database queries, and can facilitate the use of stored procedures, and its graphical user interface allows intuitive and simple system and database, it was used for storage management and maintenance in this system. In addition, due to the support of web technology, SQL Server 2012 allows users to easily publish data in the database on the Windows Application.

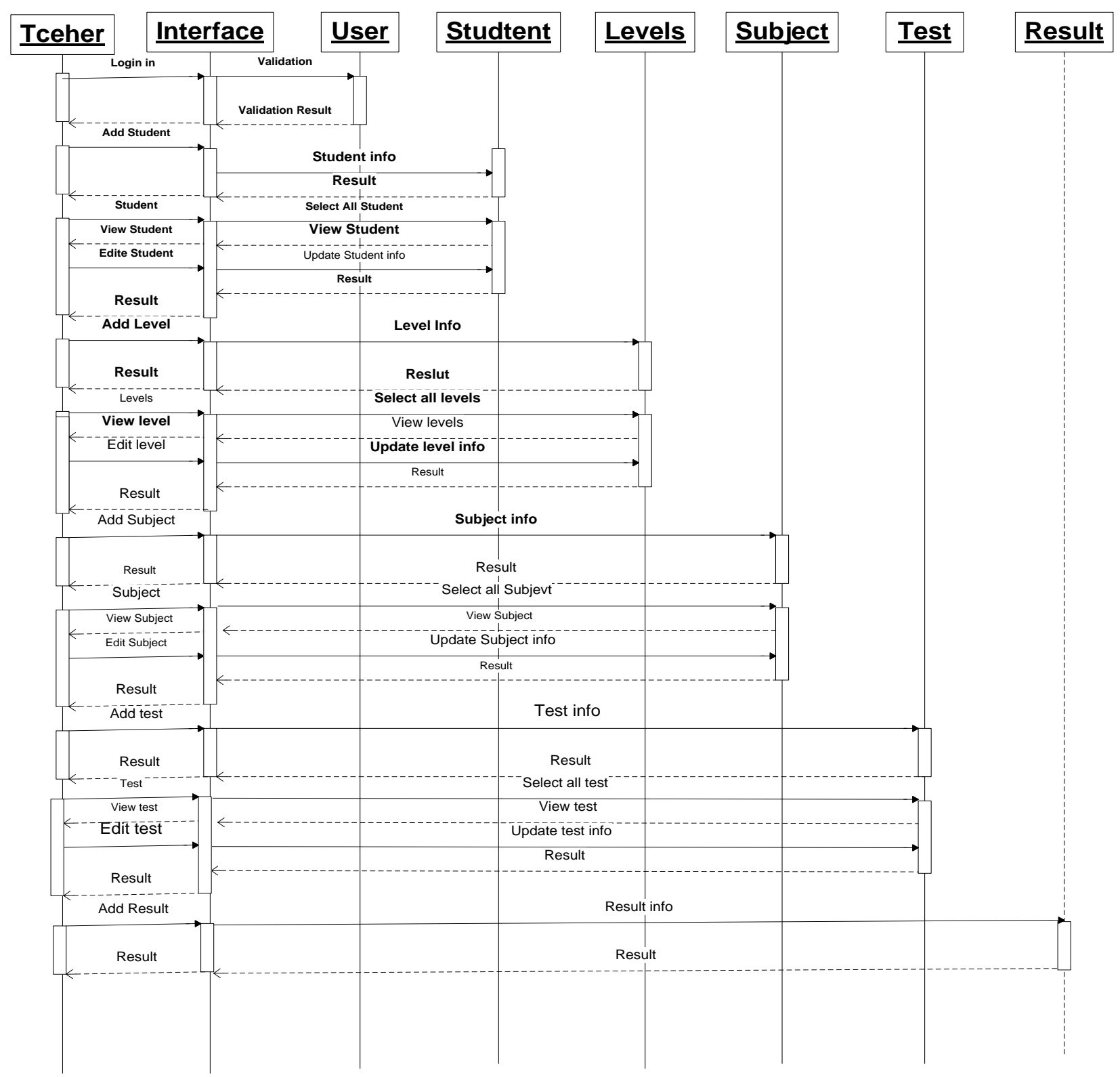

Fig. 15. Admin (teacher) sequence diagram.

\subsection{Concept Structure Design}

The concept design means that abstracting the user requirements depends on the analysis of information. This step is the key to database design. The entities and their belonging attributes involved are:

1) User (User_ID, Username, Password, Security_ID, User type, User_status, Level_ID).

2) Levels (level_ID, Level_name).

3) Questions (Id_Q, level_ID, Question). 
- Lessons: Watch (ID,VideoID, Level_id, type_lesson, lesson_name, VideoName, VideoData).

- Reading and listing (ItemID,ItemGuid, Level_id, type_lesson, lesson_name, ItemDescription, ItemImage, Itemsound).

4) Test/Answers (Q_id,Question_text, Answer_1, Answer_2, Answer_3, Answer_4, Test_id, Mark, Number_correct_answer).

5) Exam (Exam_ID, Exam_name, Level_ID, Data_exam).

6) Result (result_ID, User_ID, Grade, exam_ID).

\subsection{Designing the Database Tables}

This section describes the design of the system, the general structure of the database and the relationship between the database tables. It shows the design of the system from the point of view of the overall structure of the database and relationships of the database tables.

The database design of the system follows the system function analysis, according to the objectives of system requirements. The learning English Language system is based on Visual Studio and SQL Server which stores the most important data tables, such as Users (Teachers and Students) table, Levels Table, Questions table, Tests/Answers table, Results table. The Primary and Foreign keys in each data table represents their corresponding relationships.

Table 1. User Entity Attributes

\begin{tabular}{llll}
\hline \hline Sr.No & Field Name & Data type & Constraints \\
\hline 1 & User_id & Int & Primary Key \\
2 & Username & Nvarchar & \\
3 & Password & Nvarchar & \\
4 & Scurnum & Nvarchar & \\
5 & Id_usertype & Int & Foreign keys \\
6 & State & Int & \\
7 & Level_id & Int & Foreign keys \\
\hline \hline
\end{tabular}

\subsubsection{Entity relation diagram}

The Entity Relationship Diagrams (ERD) has been used to organize the data in the system into entities and utilized to define the relationships between these entities. This process has allowed the analyst to produce a good database structure so that the data can be stored and retrieved in a most efficient manner [3]. Fig. 16 shows the schema of the database that illustrates how the system works and how the lessons, levels, users, questions and test/answers components are represented.

The diagram also shows the tables in the Database, and how the relationships between entities are created. This diagram is created using SQL Workbench. Rectangles represent entities; the entity name is placed at the top and the attributes are at the bottom.

\subsubsection{Database tables}

The tables of the database system have also been designed, these tables are User, Levels, Questions, Lessons, Test /Answers, and Results.

\subsubsection{User entity}

Table Description: Table 1 provides information about the user like the username, password, code security, user type, states, and level Id.

The previous table contains a primary key and two foreign keys to establish the relationship with User_type table and level tables). (Fig. 17) shows the one-to-many relationship between the level and user entities (Fig. 18) shows the one-to-many relationship between the user-type and user entities. 


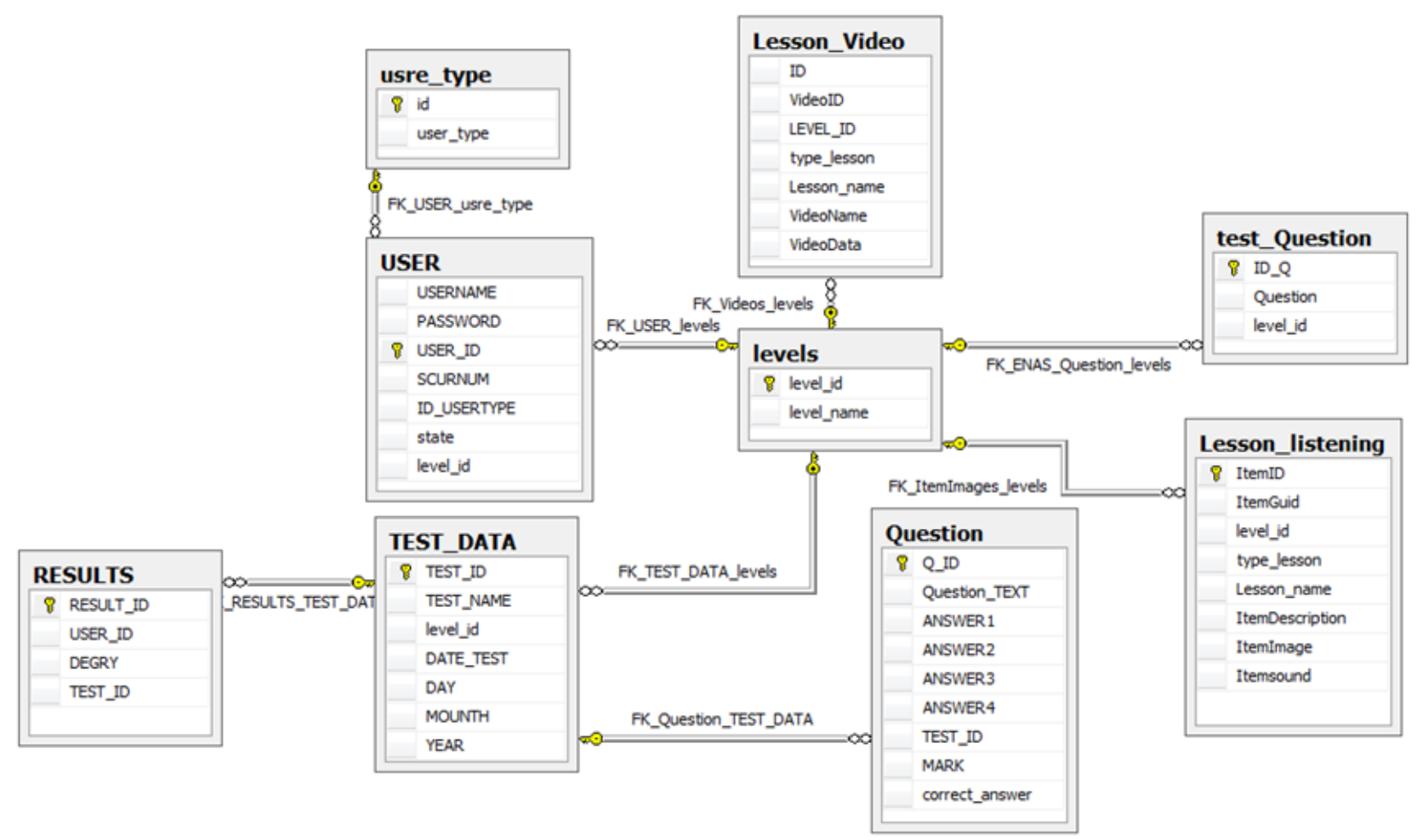

Fig. 16. Entity relation diagram.

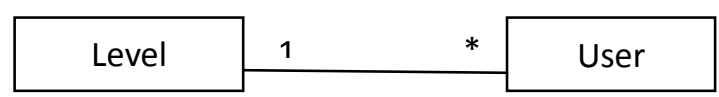

Fig. 17. Level-user relationship.

\begin{tabular}{|c|c|c|c|}
\hline User type & 1 & $*$ & User \\
\hline
\end{tabular}

Fig. 18. User_type-user relationship.

\subsubsection{Level entity}

Description: Table 2 provides information about the Level_Id, level_name attributes that belong to the Levels entity.

\subsubsection{Test_question entity}

Table Description: Table 3 provides information about the question Id, question name and level Id that belong to this entity.

Table 2. Level Entity

\begin{tabular}{cccc}
\hline \hline Sr.No & Field Name & Data type & Constraints \\
\hline 1 & level_id & Int & Primary Key \\
2 & Level_name & Nvarchar & \\
\hline \hline
\end{tabular}

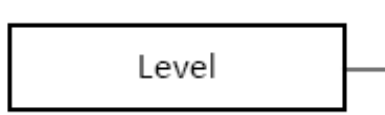

Fig. 19. Level-test_question relationship.
Table 3. Test_Question Entity

\begin{tabular}{llll}
\hline \hline Sr.No & Field & Data type & Constraints \\
\hline 1 & Name & Int & Primary Key \\
2 & Id_Q & Nvarchar & \\
3 & Question & Foreign keys \\
\hline \hline & level_id & & \\
& & & \\
& & Test_Question & \\
\end{tabular}

foreign key to indicate the relationship with the Level 
table. Fig. 19 shows the one-to-many relationship between the level and Test-Question entities.

\subsubsection{Questions entity}

Table Description: Table 4 provides information about the tests and a set of questions for each test and grades to be achieved by students.

Table 4: Qestions Entity Attributes

\begin{tabular}{llll}
\hline \hline Sr.No & Field Name & Data type & Constraints \\
\hline 1 & Id_Q & Int & Primary Key \\
2 & Question_text & Nvarchar & \\
3 & Answer1 & Nvarchar & \\
4 & Answer2 & Nvarchar & \\
5 & Answer3 & Nvarchar & \\
6 & Answer4 & Nvarchar & \\
7 & Test_id & Int & Foreign key \\
8 & MARK & Float & \\
9 & Correct_answer & Int & \\
\hline \hline
\end{tabular}

The previous table contains a primary key and a foreign key to present the relationship with the Test_Data table. Fig. 20 shows the one-to-many relationship between the Test_Data and Question entities.

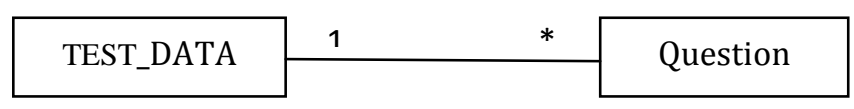

Fig. 20. Test_data-question.

\subsubsection{Test_data entity}

Table Description: Table 5 provides information about the tests to be undertaken by students.

Table 5. Test_Data Entity Attributes

\begin{tabular}{llll}
\hline \hline Sr.No & Field Name & Data type & Constraints \\
\hline 1 & TEST_ID & Int & Primary Key \\
2 & TEST_NAME & Nchar & \\
3 & Level_id & Int & Foreign key \\
4 & DATE_TEST & Datetime & \\
\hline \hline
\end{tabular}

The previous table contains a primary key and a foreign key to indicate the relationship with the Level table. Fig. 21 shows the one-to-many relationship between the level and Test-Data entities.

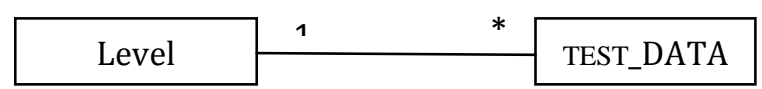

Fig. 21. Level-test_data relationship.

\subsubsection{User_type entity}

Table Description: Table 6 provides information about the user type entity.

Table 6. User_Type Entity Attributes

\begin{tabular}{llll}
\hline \hline Sr.No & Field Name & Data type & Constraints \\
\hline 1 & Id & Int & Primary Key \\
2 & User_type & Nchar & \\
\hline \hline
\end{tabular}

Table 7. Lesson_Listening Entity Attributes

\begin{tabular}{llll}
\hline \hline Sr.No & Field Name & Data type & Constraints \\
\hline 1 & ItemID & Int & Primary Key \\
2 & ItemGuid & Uniqueidentifier & \\
3 & Level_id & Int & Foreign keys \\
4 & type_lesson & Varchar & \\
5 & Lesson_name & Varchar & \\
6 & ItemDescription & Varchar & \\
7 & ItemImage & varbinary(MAX) & \\
8 & Itemsound & varbinary(MAX) & \\
\hline \hline
\end{tabular}




\subsubsection{Lesson_listening entity}

Table Description: Table 7 provides information about the lessons (listening) entity.

This table contains a primary key and a foreign key to present the relationship with the Level table. We have set up a table containing the column FILESTREAM. This column is of type VARBINARY (MAX) that contains the FILESTREAM feature enabled. The FILESTREAM column is created by adding the FILESTREAM attribute to the two columns called [ItemImage] and [itemsound] which are of type VARBINARY(MAX). Only VARBINARY (MAX) columns can be used for FILESTREAM storage. Fig. 22 shows the one-to-many relationship between the level and lesson-listening entities.

\begin{tabular}{|c|c|c|}
\hline Level & 1 & Lesson_listening \\
\hline
\end{tabular}

Fig. 22. Level-lesson_listening relationship.

\subsubsection{Lesson_videos entity}

Table Description: Table 8 provides information about the Lessons (Visual) entity.

Table 8. Lesson_Videos Entity Attributes

\begin{tabular}{llll}
\hline \hline No & Field Name & Data type & Constraints \\
\hline 1 & ID & Int & Primary Key \\
2 & VideoID & Uniqueidentifier & \\
3 & Level_id & Int & Foreign key \\
4 & type_lesson & Varchar & \\
5 & Lesson_name & Varchar & \\
6 & VideoName & Varchar & \\
7 & VideoData & varbinary(MAX) & \\
\hline \hline
\end{tabular}

Table 9. Results Attributes

\begin{tabular}{llll}
\hline \hline Sr.No & Field Name & Data type & Constraints \\
\hline 1 & Result_Id & Int & Primary Key \\
2 & User_Id & Int & \\
3 & Degry & Int & \\
4 & Test_Id & Int & Foreign key \\
\hline \hline
\end{tabular}

This table contains a primary key and a foreign key to present the relationship with the table Level. A table has been set up containing the column FILESTREAM. This column is of type VARBINARY (MAX) that contains the FILESTREAM feature enabled.

The FILESTREAM column is created by adding the FILESTREAM attribute to the column VideoData which is of type VARBINARY (MAX).

\subsubsection{Results entity}

Table Description: Table 9 provides information about the results gained by students.

This table contains a primary key and a foreign key to indicate the relationship with the result table.

Fig. 23 shows the one-to-many relationship between the Test_Data and Results entities.

\begin{tabular}{|l|ll|}
\hline Test_data & $*$ & $*$ \\
\end{tabular}

Fig. 23. Test_data-results relationship.

\section{Conclusion}

This paper has presented a novel approach to develop the English Language Learning System via utilizing the Filestream technique supported by SQL server 2012. The system proved to be successful in allowing an efficient storage and management of unstructured data (video, sound and pictures). The UML diagrams have been used successfully to achieve the analysis and design phases of the system. The outcome of this work is an attempt to maintain an efficient and effective storage and access to BLOB data available and used in the system. This will in turn allow the ELLS to operate smoothly and improve its quality and performance. This paper is one of a series of papers that will follow to present the implementation, testing, and results of the system. 


\section{References}

[1] Shaun, T. J. (2011, February). SQL Server 2008 R2 FILESTREAM Design and Implementation Considerations.

[2] Lobel, L., Andrew, J., \& Stephen, F. (2008). Programming Microsoft@ SQL Server® 2008. New York.

[3] Microsoft. (2008, July). Managing Unstructured Data with SQL Server. Retrieved June 19, 2014, from http://download.microsoft.com/download/a/c/d/acd8e043-d69b-4f09-bc9e-4168b65aaa71/SQL200 8UnstructuredData.doc

[4] Rafique, F. \& Javar, S. (2009). Implementation of Match-Making Portal (Report No. 09074). Vaxjo University, School of Mathematics and Systems Engineering.

[5] Sparx Systems. (2007). Using UML - Behavioral Modeling Diagrams. Retrieved June 23, 2014, from http://www.sparxsystems.com.au/downloads/whitepapers/UML_Tutorial_Part_2_Introduction.pdf

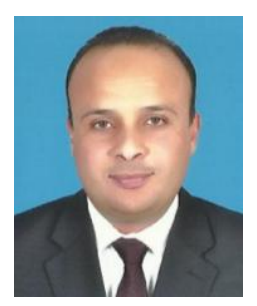

Mohamed R. Mhereeg was born in Gharian, Libya in 1977. He received his M.Sc. degree in computing from Cardiff University, Cardiff, UK in 2006; the PhD degree in computing science from the University of Glamorgan, Glamorgan, UK in 2011.

Currently, he is a lecturer at the University of Tripoli, Libya. From 2007 to 2011, he was a variable hours lecturer delivering modules at both the undergraduate and M.Sc. levels with the University of Glamorgan, UK, and he was a research data analyst in University of Swansea, UK in 2011. He was an author of the book entitled: Building an autonomous and distributed multi-agent monitoring system to provide an automatic classification of end users, Germany, LAB Lambert Academic Publishing, 2013. He has a number of published articles like: The development of the multi-agent classification system (MACS) in compliance with FIPA specifications, The design of the multi-agent classification system (MACS), The implementation of the multi-agent classification system (MACS) in compliance with FIPA specifications.

Dr. Mhereeg is a member in the Fulbright International Exchange Alumni, an author in the IEEE Computer Society, and an author in the ACM Digital Library. He is also the owner of the Fulbright Visiting Scholar Program Award.

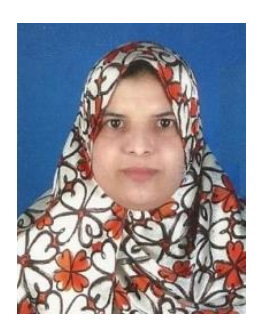

Asma G. Tawil was born in Janzoor, Libya in 1981. She received her M.Sc. degree in information technology from the Libyan Academy for Postgraduate Studies, Tripoli, Libya in 2015.

Currently, she is a lecturer at the Faculty of Engineering Technology, Institute of Computing Technology. She has a past experience as a software engineer and researcher specializing in the research, design, development and analysis of object oriented software systems. She also has experience in the full lifecycle of software production including requirements definition, prototyping, design, implementation, testing and maintenance. She is an expert trainer in microsoft applications and in a decent variety of programming languages, databases, and web applications design. Dr. Tawil has been awarded a scholarship by the Libyan Government to undertake a PhD degree in information technology in Germany. 